\title{
A NON-UNITAL GENERALIZED TRACE AND LINEAR COMPLEX STRUCTURES
}

\author{
ADAM COFFMAN
}

Abstract. A basis-free formula for the generalized trace of a linear map between tensor products of vector spaces is proposed which does not refer to scalar multiplication or scalar valued functions. The main application is to real vector spaces with complex structure operators.

Mathematics subject classification (2020): Primary 15A69; Secondary 15A15, 18M10.

Keywords and phrases: Generalized trace, monoidal category, complex structure, real structure.

\section{REFERENCES}

[1] F. Anderson And K. Fuller, Rings and Categories of Modules, GTM 13, Springer-Verlag, New York, 1974. MR0417223 (54 \#5281)

[2] S. Awodey, Category Theory, 2nd ed., OLG 52, Oxford, 2010. MR2668552 (2011g:18001)

[3] J. BLINN, Algebraic Geometry for Computer Graphics, University of Washington lecture notes, 2013, https://courses.cs.washington.edu/courses/cse590b/13au/.

[4] N. Bourbaki, Algebra I, Chapters 1-3, Hermann, Paris, 1974. MR0354207 (50 \#6689)

[5] B. Coecke And A. Kissinger, Picturing Quantum Processes - A First Course in Quantum Theory and Diagrammatic Reasoning, Cambridge Univ. Press, 2017.

[6] A. Coffman, Notes on Abstract Linear Algebra, unpublished notes, http://users.pfw.edu/CoffmanA/.

[7] P. Etingof, S. Gelaki, D. Nikshych, And V. Ostrik, Tensor Categories, SURV 205, AMS, 2015. MR3242743

[8] K. FilipiaK, D. KLein, And E. VojtKovÁ, The properties of partial trace and block trace operators of partitioned matrices, Electronic Journal of Linear Algebra 33 (2018), 3-15. MR3826862

[9] A. Joyal, R. Street, And D. Verity, Traced monoidal categories, Math. Proc. Camb. Phil. Soc. (3) 119 (1996), 447-468. MR1357057 (96m:18014)

[10] X. LU, Y. YE, AND S. HU, A graphical calculus for semi-groupal categories, Appl. Categ. Structures (2) 27 (2019), 163-197. MR3925574

[11] G. Maltsiniotis, Traces dans les catégories monoïdales, dualité et catégories monoüdales fibrées, Cahiers Topologie Géom. Différentielle Catég. (3) 36 (1995), 195-288. MR1352535 (97d:18007)

[12] K. Ponto And M. Shulman, Shadows and traces in bicategories, J. Homotopy Relat. Struct. (2) 8 (2013), 151-200. MR3095324

[13] K. Ponto And M. Shulman, Traces in symmetric monoidal categories, Expo. Math. (3) 32 (2014), 248-273. MR3253568

[14] J. Richter-Gebert And P. Lebmeir, Diagrams, tensors and geometric reasoning, Discrete Comput. Geom. (2) 42 (2009), 305-334. MR2519881 (2011c:51018)

[15] D. Salamon, Spin Geometry and Seiberg-Witten Invariants, ETH-Zürich preprint, August 1999, http://people.math.ethz.ch/ ${ }^{\sim}$ salamon/PREPRINTS/witsei.pdf.

[16] S. Stolz And P. Teichner, Traces in monoidal categories, Trans. Amer. Math. Soc. (8) 364 (2012), 4425-4464. MR2912459

[17] C. VAfA, E. ZASLOw, et al, eds., Mirror Symmetry, Clay Math. Monog. 1, AMS, 2003. MR2003030 (2004g:14042) 